\title{
On Holomorphic Curves Tangent to Real Hypersurfaces of Infinite Type
}

\author{
Joe Kamimoto ${ }^{1}$ (D)
}

Received: 13 August 2020 / Accepted: 12 November 2020 / Published online: 5 December 2020

(c) The Author(s) 2020

\section{Abstract}

The purpose of this paper is to investigate the geometric properties of real hypersurfaces of D'Angelo infinite type in $\mathbb{C}^{n}$. In order to understand the situation of flatness of these hypersurfaces, it is natural to ask whether there exists a nonconstant holomorphic curve tangent to a given hypersurface to infinite order. A sufficient condition for this existence is given by using Newton polyhedra, which is an important concept in singularity theory. More precisely, equivalence conditions are given in the case of some model hypersurfaces.

Keywords Holomorphic curve · Real hypersurface · D'Angelo type ·

Bloom-Graham type $\cdot$ Infinite type

Mathematics Subject Classification 32F18 (32T25)

\section{Introduction}

Let $M$ be a ( $C^{\infty}$ smooth) real hypersurface in $\mathbb{C}^{n}$ and let $p$ lie on $M$. Let $r$ be a local defining function for $M$ near $p(\nabla r \neq 0$ when $r=0)$. In [6,7], the following invariant is introduced:

$$
\Delta_{1}(M, p):=\sup _{\gamma \in \Gamma} \frac{\operatorname{ord}(r \circ \gamma)}{\operatorname{ord}(\gamma-p)}
$$

where $\Gamma$ denotes the set of (germs of) nonconstant holomorphic mappings $\gamma$ : $(\mathbb{C}, 0) \rightarrow\left(\mathbb{C}^{n}, p\right)$. (For a $C^{\infty}$ mapping $h: \mathbb{C} \rightarrow \mathbb{C}$ or $\mathbb{C}^{n}$ such that $h(0)=0$, let ord $(h)$ denote the order of vanishing of $h$ at 0 .) The invariant $\Delta_{1}(M, p)$ is called the D'Angelo type of $M$ at $p$. We say that $M$ is of finite type at $p$ if $\Delta_{1}(M, p)<\infty$

\footnotetext{
$凶 \quad$ Joe Kamimoto

joe@math.kyushu-u.ac.jp

1 Faculty of Mathematics, Kyushu University, Motooka 744, Nishi-ku, Fukuoka 819-0395, Japan
} 
and of infinite type at $p$ otherwise (the latter case will be denoted by $\Delta_{1}(M, p)=\infty$ ). The class of finite type plays crucial roles in the study of the local regularity in the $\bar{\partial}$ Neumann problem over pseudoconvex domains $\Omega$ with smooth boundary $\partial \Omega$. Indeed, it was shown by Catlin [4,5] that $M=\partial \Omega$ is of finite type at $p$ if and only if a local subelliptic estimate at $p$ holds. From its importance, real hypersurfaces of finite type have been deeply investigated from various points of view.

On the other hand, to understand the geometric properties of real hypersurfaces of infinite type is also an interesting subject in the study of several complex variables. These hypersurfaces contain some kind of strong flatness. In order to describe the geometric structure of this flatness, the situation of contact of holomorphic curves with the respective hypersurface must be carefully observed. In this paper, we mainly consider the following question:

Question 1 When does there exist a nonconstant holomorphic curve $\gamma_{\infty}$ tangent to $M$ at $p$ to infinite order?

Since the condition of the desired curve $\gamma_{\infty}$ in Question 1 can be written as

$$
\left(r \circ \gamma_{\infty}\right)(t)=O\left(t^{N}\right) \quad \text { as } t \in \mathbb{C} \rightarrow 0 \text {, for every } N \in \mathbb{N},
$$

the condition $\Delta_{1}(M, p)=\infty$ is necessary for the existence of the curve $\gamma_{\infty}$. It has been shown in [7,11,19] that when $M$ is real analytic, the above two conditions are equivalent (in this case, the curve $\gamma_{\infty}$ is contained in $M$ ). Moreover, in the case of smooth $M$, this equivalence is also shown in the formal series sense in $[8,16]$. But, in general, the sufficient direction is not true. Indeed, the nonexistence of the curve $\gamma_{\infty}$ in (1.2) is shown in the case of some real hypersurfaces constructed in $[3,9,16,22]$. Understanding flatness on hypersurfaces of infinite type has been recognized to be a delicate issue.

In this paper, in order to investigate the flatness of real hypersurfaces, we use not only holomorphic curves but also Newton polyhedra of defining functions, which plays important roles in singularity theory (cf. [1,2]). Approach from the viewpoint of singularity theory is useful in the study of types and there have been many works of the sort $([10,11,13,15,20]$, etc. $)$.

First, we consider the relationship among many kinds of infinite type hypersurfaces. Let $\Gamma^{\text {reg }}:=\{\gamma \in \Gamma: \operatorname{ord}(\gamma)=1\}$ and define $\Delta_{1}^{\text {reg }}(M, p):=\sup _{\gamma \in \Gamma^{\operatorname{reg}}}\{\operatorname{ord}(r \circ \gamma)\}$, which is called regular type of $M$ at $p$. Note that $\Delta_{1}^{\text {reg }}(M, p) \leq \Delta_{1}(M, p)$.

Proposition 1.1 Let us consider the following eight conditions for a real hypersurface $M$ at $p$ :

(1) $\Delta_{1}(M, p)=\infty$;

(2) $\Delta_{1}^{\mathrm{reg}}(M, p)=\infty$;

(3) There exists a $\gamma \in \Gamma^{\mathrm{reg}}$ tangent to $M$ at $p$ to infinite order;

(4) There exists a $\gamma \in \Gamma$ tangent to $M$ at $p$ to infinite order;

(5) There exists a holomorphic coordinate $(z)=\left(z_{1}, \ldots, z_{n}\right)$ at $p$ such that $p=0$ and a defining function $r$ for $M$ on $(z)$ is not convenient (see Sect. 2); 
(6) There exists a holomorphic coordinate (z) at $p$ such that $p=0$ and the Newton polyhedron of a defining function for $M$ on (z) (see Sect. 2) takes the form $\mathcal{N}_{+}(r)=\left\{\left(\xi_{1}, \ldots, \xi_{n}\right) \in \mathbb{R}_{+}^{n}: \xi_{n} \geq 1\right\}$

(7) The Bloom-Graham type of $M$ at $p$ is infinity (i.e. there are complex submanifolds of codimension one tangent to $M$ at $p$ to arbitrarily higher order);

(8) $M$ is Levi-flat near $p$.

Then, among the above eight conditions, the following implications hold:

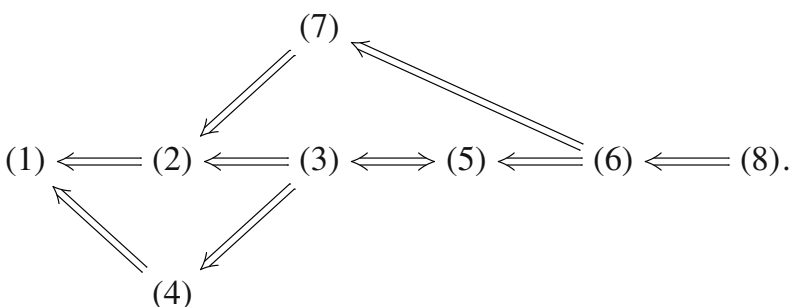

The proof of the above proposition will be given in Sect. 4.1 .

Remark 1.2 In the above proposition, for each implication with only one direction, its opposite direction is not true (see Remark 4.2 in Sect. 4 for details).

The following theorem gives a sufficient condition for the existence of the curve $\gamma_{\infty}$ in Question 1. This condition is described by using Newton polyhedra of defining functions for real hypersurfaces.

Theorem 1.3 If there exists an $\mathcal{N}$-canonical coordinate (z) for $M$ at $p$, then the five conditions (1)-(5) are equivalent.

The definition of $\mathcal{N}$-canonical coordinates will be given in Sect. 2 (Definition 2.3). The proof of the above theorem will be given in Sect. 4.2.

The following corollary can be directly obtained from Theorem 1.3.

Corollary 1.4 Suppose that $\Delta_{1}(M, p)=\infty$. If there is no $\gamma \in \Gamma$ tangent to $M$ at $p$ to infinite order, then $M$ does not admit any $\mathcal{N}$-canonical coordinates at $p$.

It is easy to check that the examples of hypersurface constructed in $[3,9,16,22]$ do not admit any $\mathcal{N}$-canonical coordinates. More exactly, we will give equivalence conditions in more restricted cases in Sects. 5 and 6.

Next, it is seen in [15] that when $M$ is the boundary of pseudoconvex Reinhardt domains, $M$ always admits an $\mathcal{N}$-canonical coordinate. Therefore, Theorem 1.3 implies the following.

Corollary 1.5 Let M be the boundary of pseudoconvex Reinhardt domains (with smooth boundary) and let $p$ lie in $M$. If $\Delta_{1}(M, p)=\infty$, then there exists a $\gamma \in \Gamma^{\text {reg }}$ tangent to $M$ at $p$ to infinite order. 
This paper is organized as follows. In Sect. 2, we recall the concepts: Newton polyhedra, $\mathcal{N}$-nondegeneracy condition and $\mathcal{N}$-canonical coordinates, which were introduced in [15]. In Sect. 3, for the analysis later, we prepare appropriate coordinates on which hypersurfaces are expressed in clear form. Section 4 is devoted to the proof of results given in the Introduction. More precise results are given in the two dimensional case in Sect. 5 and the higher dimensional case under the Bloom-Graham infinity type assumption in Sect. 6. Since the Bloom-Graham type is the same as the D'Angelo type in the two-dimensional case, some results in Sect. 5 can be considered as special cases of those in Sect. 6. But, they are separately explained to make clear their difference. Lastly, we consider open problems in Sect. 7.

Notation, symbols and terminology.

- We denote $\mathbb{Z}_{+}:=\{n \in \mathbb{Z}: n \geq 0\}$ and $\mathbb{R}_{+}:=\{x \in \mathbb{R}: x \geq 0\}$.

- The multi-indices are used as follows. For $z=\left(z_{1}, \ldots, z_{n}\right), \bar{z}=\left(\bar{z}_{1}, \ldots, \bar{z}_{n}\right), \in$ $\mathbb{C}^{n}, \alpha=\left(\alpha_{1}, \ldots, \alpha_{n}\right), \beta=\left(\beta_{1}, \ldots, \beta_{n}\right) \in \mathbb{Z}_{+}^{n}$, define

$$
\begin{aligned}
& z^{\alpha}:=z_{1}^{\alpha_{1}} \cdots z_{n}^{\alpha_{n}}, \bar{z}^{\beta}:=\bar{z}_{1}^{\beta_{1}} \cdots \bar{z}_{n}^{\beta_{n}}, \\
& |\alpha|:=\alpha_{1}+\cdots+\alpha_{n}, \quad \alpha !:=\alpha_{1} ! \cdots \alpha_{n} !, \quad 0 !:=1, \\
& D^{\alpha}:=\frac{\partial^{|\alpha|}}{\partial z_{1}^{\alpha_{1}} \cdots \partial z_{n}^{\alpha_{n}}}, \quad \bar{D}^{\beta}:=\frac{\partial^{|\beta|}}{\partial \bar{z}_{1}^{\beta_{1}} \cdots \partial \bar{z}_{n}^{\beta_{n}}} .
\end{aligned}
$$

- We always consider smooth functions, mappings, real hypersurfaces and complex curves as their respective germs without any mentioning. The following rings of germs of $\mathbb{C}$-valued functions are considered:

- $C_{0}^{\infty}\left(\mathbb{C}^{n}\right)$ is the ring of germs of $C^{\infty}$ functions at the origin in $\mathbb{C}^{n}$.

- $\mathcal{O}_{0}(\mathbb{C})$ is the ring of germs of holomorphic functions at the origin in $\mathbb{C}$.

- We always take a good parametrization for corves $\gamma \in \Gamma$ without any mentioning. That is to say, a point on the curve, defined by $t \mapsto \gamma(t)$, corresponds to only one value of $t$. For example, $\left(t, t^{2}\right)$ is good, but $\left(t^{2}, t^{4}\right)$ is not good.

- We use the words pure terms for any harmonic polynomial and mixed terms for any sum of monomials that are neither holomorphic nor anti-holomorphic.

\section{Newton Polyhedra for Real Hypersurfaces}

Let us define the Newton polyhedron of a real-valued smooth function $F$ defined near the origin in $\mathbb{C}^{n}$. The Taylor series expansion of $F$ at the origin is

$$
F(z, \bar{z}) \sim \sum_{\alpha, \beta \in \mathbb{Z}_{+}^{n}} C_{\alpha \beta} z^{\alpha} \bar{z}^{\beta} \quad \text { with } C_{\alpha \beta}=\frac{1}{\alpha ! \beta !} D^{\alpha} \bar{D}^{\beta} F(0,0)
$$


The Newton polyhedron of $F$ is defined by

$$
\mathcal{N}_{+}(F)=\text { The convex hull of }\left(\bigcup_{\alpha+\beta \in S(F)}\left(\alpha+\beta+\mathbb{R}_{+}^{n}\right)\right) \text {, }
$$

where $S(F)=\left\{\alpha+\beta \in \mathbb{Z}_{+}^{n}: C_{\alpha \beta} \neq 0\right\}$. The Newton diagram $\mathcal{N}(F)$ of $F$ is defined to be the union of the compact faces of $\mathcal{N}_{+}(F)$. We use coordinates $(\xi)=\left(\xi_{1}, \ldots, \xi_{n}\right)$ for points in the plane containing the Newton polyhedron. The following classes of functions $F$ simply characterized by using their Newton polyhedra often appear in this paper:

- $F$ is called to be flat at 0 if $\mathcal{N}_{+}(F)$ is an empty set.

- $F$ is called to be convenient at 0 if $\mathcal{N}_{+}(F)$ meets every coordinate axis.

Let $(z)=\left(z_{1}, \ldots, z_{n}\right)$ be a holomorphic coordinate around $p$ such that $p=0$. Let $r$ be a local defining function for $M$ near $p$ on the coordinate $(z)$. For a given tuple $(M, p ;(z))$, we define a quantity $\rho_{1}(M, p ;(z)) \in \mathbb{Z}_{+} \cup\{\infty\}$ as follows. If $r$ is convenient, then let

$$
\rho_{1}(M, p ;(z)):=\max \left\{\rho_{j}(r): j=1, \ldots, n\right\},
$$

where $\rho_{j}(r)$ is the coordinate of the point at which the Newton diagram $\mathcal{N}(r)$ intersects the $\xi_{j}$-axis. Otherwise, let $\rho_{1}(M, p ;(z)):=\infty$. We remark that $\rho_{1}(M, p ;(z))$ depends on the chosen coordinate $(z)$, but it is independent of the choice of defining functions after fixing a coordinate. Considering the curves $\gamma_{j}(t)=\left(0, \ldots,{ }_{t}^{(j)}\right.$ $, \ldots, 0) \in \Gamma^{\mathrm{reg}}$ for $j=1, \ldots, n$, we can see that the inequality $\rho_{1}(M, p ;(z)) \leq$ $\Delta_{1}^{\mathrm{reg}}(M, p)$ always holds.

Next, let us introduce an important concept " $\mathcal{N}$-nondegeneracy condition" on a smooth function $F$ defined near the origin in $\mathbb{C}^{n}$.

Let $\kappa$ be a compact face of $\mathcal{N}_{+}(F)$. The $\kappa$-part of $F$ is the polynomial defined by

$$
F_{\kappa}(z, \bar{z})=\sum_{\alpha+\beta \in \kappa} C_{\alpha \beta} z^{\alpha} \bar{z}^{\beta}
$$

The set of holomorphic curves $\Gamma_{\kappa}$ is defined by

$$
\Gamma_{\kappa}:=\left\{\left(c_{1} t^{a_{1}}, \ldots, c_{n} t^{a_{n}}\right): c \in(\mathbb{C} \backslash\{0\})^{n}, t \in \mathbb{C}, a \in \mathbb{N}^{n} \text { determines } \kappa\right\},
$$

where $c=\left(c_{1}, \ldots, c_{n}\right) \in(\mathbb{C} \backslash\{0\})^{n}, a=\left(a_{1}, \ldots, a_{n}\right) \in \mathbb{N}^{n}$ and " $a \in \mathbb{N}^{n}$ determines $\kappa$ " means that the set $\left\{\xi \in \mathcal{N}_{+}(F): \sum_{j=1}^{n} a_{j} \xi_{j}=l\right\}$ coincides with the face $\kappa$ for some $l \in \mathbb{N}$.

Definition 2.1 Let $\kappa$ be a compact face of $\mathcal{N}_{+}(F)$. The $\kappa$-part $F_{\kappa}$ of $F$ is said to be $\mathcal{N}$-nondegenerate if

$$
F_{\kappa} \circ \gamma \not \equiv 0 \text { for any } \gamma \in \Gamma_{\kappa} \text {. }
$$


A function $F$ is said to be $\mathcal{N}$-nondegenerate, if $F_{\kappa}$ is $\mathcal{N}$-nondegenerate for every compact face $\kappa$ of $\mathcal{N}_{+}(F)$.

The above concept is analogous to the nondegeneracy condition introduced by Kouchnirenko [18], which plays important roles in the singularity theory. Detailed properties of this condition are explained in [15].

Definition 2.2 A holomorphic coordinate ( $z$ ) at $p$ is said to be $\mathcal{N}$-canonical for $M$ at $p$ if a local defining function $r$ for $M$ on $(z)$ is $\mathcal{N}$-nondegenerate.

The following relationship

$$
\Delta_{1}(M, p) \geq \Delta_{1}^{\mathrm{reg}}(M, p) \geq \rho_{1}(M, p ;(z))
$$

is always established for every coordinate $(z)$ at $p$. The following theorem shows that the equalities in (2.3) are satisfied under the $\mathcal{N}$-nondegeneracy condition.

Theorem 2.3 [15] If there exists an $\mathcal{N}$-canonical coordinate $(z)$ at $p$, then the following equalities hold:

$$
\Delta_{1}(M, p)=\Delta_{1}^{\mathrm{reg}}(M, p)=\rho_{1}(M, p ;(z))
$$

Note that the above theorem is valid for the infinite type case. From the above theorem, the existence of $\mathcal{N}$-canonical coordinates implies that both values of $\Delta_{1}(M, p)$ and $\Delta_{1}^{\mathrm{reg}}(M, p)$ can be directly seen from geometrical Newton data of $M$ at $p$.

\section{Standard Coordinates}

Let $M$ be a real hypersurface in $\mathbb{C}^{n+1}(n \geq 1)$ and let $p$ lie in $M$.

It follows from Taylor's formula that there exists a holomorphic coordinate $(z, w):=\left(z_{1}, \ldots, z_{n}, w\right)$ at $p$ on which a local defining function $r$ for $M$ is expressed near the origin as in the following form:

$$
r(z, w, \bar{z}, \bar{w})=2 \operatorname{Re}(w)+F(z, \bar{z})+R_{1}(z, \bar{z}) \cdot \operatorname{Im}(w)+R_{2}(z, w, \bar{z}, \bar{w}),
$$

where

(i) $F \in C_{0}^{\infty}\left(\mathbb{C}^{n}\right)$ satisfies that $F(0,0)=0$ and $|\nabla F(0,0)|=0$;

(ii) $R_{1} \in C_{0}^{\infty}\left(\mathbb{C}^{n}\right)$ and $R_{2} \in C_{0}^{\infty}\left(\mathbb{C}^{n+1}\right)$. Moreover, $R_{1}, R_{2}$ satisfies that $\operatorname{ord}\left(R_{1}\right) \geq$ 1 and $\left|R_{2}\right| \leq C|\operatorname{Im}(w)|^{2}$ near $(z, w)=0$ where $C$ is a positive constant independent of $(z, w)$.

Of course, there may be many such coordinates, which are said to be standard for $M$ at $p$.

Furthermore, if there exists a holomorphic coordinate $(z, w):=\left(z_{1}, \ldots, z_{n}, w\right)$ around $p$ on which a local defining function $r$ for $M$ is expressed near the origin as in the model form:

$$
r(z, w, \bar{z}, \bar{w})=2 \operatorname{Re}(w)+F(z, \bar{z}),
$$


where $F$ is as in (3.1), then $(z, w)$ is called a good (standard) coordinate for $M$ at $p$. Note that good coordinates do not always exist for all hypersurfaces.

\section{Proofs of Results in the Introduction}

Let $M$ be a real hypersurface in $\mathbb{C}^{n}$ and let $p$ lie in $M$.

From Theorem 2.3, under the $\mathcal{N}$-nondegeneracy condition, a defining function for $M$ is convenient if and only if $M$ is of finite type at $p$. Roughly speaking, when $M$ does not satisfy the convenience condition, $M$ contains a flat part in one direction. The following lemma exactly explains such situation.

Lemma 4.1 Let $r$ be a local defining function for $M$ near $p$ on some holomorphic coordinate $(z)$. Then the following four conditions are equivalent:

(i) $r$ is not convenient (i.e. $\left.\rho_{1}(M, p ;(z))=\infty\right)$;

(ii) There exists $k \in\{1, \ldots, n\}$ such that $\mathcal{N}_{+}(r)$ does not intersect $\xi_{k}$-axis;

(iii) There exists $k \in\{1, \ldots, n\}$ such that the Taylor series of $r$ does not contain any term consisting of $z_{k}$ and $\bar{z}_{k}$ only;

(iv) There exists $k \in\{1, \ldots, n\}$ such that $\left(r \circ \gamma_{k}\right)(t)=O\left(t^{N}\right)$ for every $N \in \mathbb{N}$, where $\gamma_{k}=(0, \ldots, \stackrel{(k)}{t}, \ldots, 0) \in \Gamma^{\mathrm{reg}}$.

The following lemma expresses a property of flat hypersurfaces by using the language of Newton polyhedra.

Lemma 4.2 The following two conditions are equivalent:

(i) There exists a standard coordinate for $M$ at $p$ such that $F$ in (3.1) is flat at the origin;

(ii) There exists a holomorphic coordinate (z) at $p$ such that $p=0$ and the Newton polyhedron of a defining function $r$ for $M$ on $(z)$ takes the form: $\mathcal{N}_{+}(r)=$ $\left\{\left(\xi_{1}, \ldots, \xi_{n}\right) \in \mathbb{R}_{+}^{n}: \xi_{n} \geq 1\right\}$.

Since the above two lemmas are easy, their proofs are omitted.

\subsection{Proof of Proposition 1.1}

(3) $\Longleftrightarrow$ (5) was stated in Lemma 4.1. (6) $\Longrightarrow$ (7) is easy to see from Lemma 4.2. (7) $\Longrightarrow(2)$ is shown in [9]. (Lemma 5 in [9] states (7) $\Longrightarrow(1)$, but its proof actually implies the above stronger implication.) The other implications are obvious.

Remark 4.3 Let us consider the converse of each implication in Proposition 1.1. We give an example of the hypersurface showing that the converse does not hold in the implication with only one direction. All our examples of hypersurface $M$ in $\mathbb{C}^{n+1}$ $(n \in \mathbb{N})$ are pseudoconvex and admit a good coordinate, i.e., $M$ is locally expressed as in the model form: $2 \operatorname{Re}(w)+F(z, \bar{z})=0$, as in (3.2). Thus, we only write a function $F(z, \bar{z})$ for each case. In the two-dimensional case, the converses of some implications are also true (see Sect. 5). In these cases, counterexamples must be constructed in $\mathbb{C}^{n}$ with $n \geq 3$. 
- $((1) \nRightarrow(2)) \quad F(z, \bar{z})=\left|z_{1}^{3}-z_{2}^{2}\right|^{2}+\left|z_{3}\right|^{2}+\cdots+\left|z_{n}\right|^{2} \quad(n \geq 2)$;

- $((2) \nRightarrow(3)) \quad F(z, \bar{z})=f\left(z_{1}, \bar{z}_{1}\right)+\left|z_{2}\right|^{2}+\cdots+\left|z_{n}\right|^{2} \quad(n \in \mathbb{N})$;

- $((1) \nRightarrow(4)) \quad F(z, \bar{z})=f\left(z_{1}, \bar{z}_{1}\right)+\left|z_{2}\right|^{2}+\cdots+\left|z_{n}\right|^{2} \quad(n \in \mathbb{N})$;

- $((4) \nRightarrow(3)) \quad F(z, \bar{z})=\left|z_{1}^{3}-z_{2}^{2}\right|^{2}+\left|z_{3}\right|^{2}+\cdots+\left|z_{n}\right|^{2} \quad(n \geq 2)$;

- $((5) \nRightarrow(6)) \quad F(z, \bar{z})=\left|z_{n}\right|^{2} \quad(n \geq 2)$;

- $((2) \nRightarrow(7)) \quad F(z, \bar{z})=\left|z_{n}\right|^{2} \quad(n \geq 2)$;

- $((7) \nRightarrow(6)) \quad F(z, \bar{z})=f\left(z_{n}, \bar{z}_{n}\right) \quad(n \in \mathbb{N})$;

- $((6) \nRightarrow(8)) \quad F(z, \bar{z})=\exp \left(-\left|z_{n}\right|^{-2}\right) \quad(n \in \mathbb{N})$.

Here $f \in C_{0}^{\infty}(\mathbb{C})$ is a subharmonic function admitting a divergent Taylor series at the origin. It was constructed in [9] (see Remark 5.8).

It is shown in $[16,22]$ (see also Corollary 6.4 in this paper) that the second example shows $(2) \nRightarrow(3)$ and $(1) \nRightarrow(4)$ in the two-dimensional case. The higher dimensional case can be easily shown. It is easy to check that the other hypersurfaces are counterexamples.

\subsection{Proof of Theorem 1.3}

It suffices to show the implication: $(1) \Longrightarrow(5)$.

Let $(z)$ be an $\mathcal{N}$-canonical coordinate for $M$ at $p$ and let $r$ be a local defining function for $M$ near $p$ on the coordinate $(z)$. From Theorem 2.3, the condition (1) implies $\rho_{1}(M, p ;(z))=\infty$, which is equivalent to the condition (5) from Lemma 4.1.

\section{Two Dimensional Case}

In this section, we more precisely consider Question 1 in the Introduction in the case when a real hypersurface $M$ is in $\mathbb{C}^{2}$. Let $p \in M$.

In the two dimensional case, many implications in Proposition 1.1 can be refined by equivalences.

Proposition 5.1 Let $M$ be a real hypersurface in $\mathbb{C}^{2}$ and let $p$ lie in $M$. Among the above eight conditions for $M$ at $p$ in Proposition 1.1, the following implications hold:

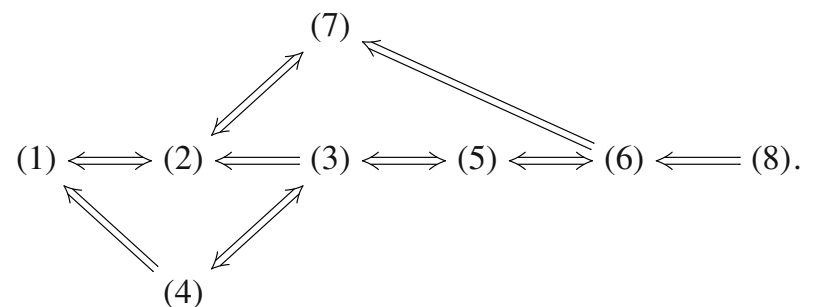

Proof $(5) \Longrightarrow(6)$ is obvious. It is known in [7] that (1), (2), (7) are equivalent. (4) $\Longrightarrow$ (3) will be shown in Lemma 5.2, below.

Lemma 5.2 If $\gamma \in \Gamma$ is tangent to $M \subset \mathbb{C}^{2}$ at $p$ to infinite order, then $\gamma \in \Gamma^{\mathrm{reg}}$. 
Proof Let $(z, w)$ be a standard coordinate for $M$ at $p$ such that $M$ is expressed as in (3.1).

First, if $F$ is flat at 0 , then the curve $\gamma$, satisfying $(r \circ \gamma)(t)=O\left(t^{N}\right)$ for every $N \in \mathbb{N}$, essentially takes the form: $\gamma(t)=(t, 0)$. This curve is regular.

Next, let us consider the case when ord $(F)=m$ with some $m \in \mathbb{N}$. In this case, the Newton polyhedron of $r$ takes the form $\mathcal{N}_{+}(r)=\left\{\xi \in \mathbb{R}_{+}^{2}: \xi_{1} / m+\xi_{2} \geq 1\right\}$. Let $\kappa:=\left\{\xi \in \mathbb{R}_{+}^{2}: \xi_{1} / m+\xi_{2}=1\right\}$, which is the only compact facet of $\mathcal{N}_{+}(r)$. If $r_{\kappa}$ were $\mathcal{N}$-nondegenerate, then Theorem 2.3 implies $\Delta_{1}(M, p)=m<\infty$, which is a contradiction. If $r_{\kappa}$ is not $\mathcal{N}$-nondegenerate, then a desired curve must be written in the form $\gamma(t)=\left(t, c t^{m}+O\left(t^{m+1}\right)\right)$ with $c \neq 0$ from the definition of the $\mathcal{N}$ nondegeneracy. This curve is also regular.

From Theorem 2.3, we can see the following.

Theorem 5.3 Let $M \subset \mathbb{C}^{2}$. If there exists an $\mathcal{N}$-canonical coordinate for $M$ at $p$, then the seven conditions (1)-(7) in Proposition 5.1 are equivalent.

The following lemma is essentially the same as Theorem 2 in [16] (see also [17]).

Lemma 5.4 Let $M \subset \mathbb{C}^{2}$. If $\Delta_{1}(M, p)=\infty$, then the Taylor series of $F$ in (3.1) at the origin consists of only pure terms on every standard coordinate.

Proof Let us assume the existence of a standard coordinate $(z, w)$ on which the Taylor series of $F$ contains a mixed term. Let $m$ be the minimum order of the mixed terms of Taylor series of $F$ and let $p_{m}(z, \bar{z})$ denote the sum of the mixed terms of order $m$.

It is easy to construct a standard coordinate $\left(z, w^{*}\right)$ on which $M$ can be expressed by the equation:

$$
2 \operatorname{Re}\left(w^{*}\right)+p_{m}(z, \bar{z})+Q(z, \bar{z})+R_{1}^{*} \cdot \operatorname{Im}\left(w^{*}\right)+R_{2}^{*}=0,
$$

where $Q \in C_{0}^{\infty}(\mathbb{C})$ with $\operatorname{ord}(Q) \geq m+1$ and $R_{1}^{*}, R_{2}^{*}$ have the same properties as those of $R_{1}, R_{2}$ in (3.1), respectively. Since $2 \operatorname{Re}\left(w^{*}\right)+p_{m}(z)$ is $\mathcal{N}$-nondegenerate, the above $\left(z, w^{*}\right)$ is $\mathcal{N}$ - canonical for $M$ at $p$. It follows from Theorem 2.3 that $\Delta_{1}(M, p)=m<\infty$, which is a contradiction.

From the above lemma, when $\Delta_{1}(M, p)=\infty$, the Taylor series of $F$ can be expressed as $2 \sum_{j=2}^{\infty} \operatorname{Re}\left(c_{j} z^{j}\right)$ where $c_{j} \in \mathbb{C}$. When the hypersurface $M$ and $p \in M$ are fixed, the sequence of complex numbers $\left\{c_{j}\right\}_{j \in \mathbb{N}}$ is determined by the chosen standard coordinates $(z, w)$ only. For a given coordinate $(z, w)$, we define the formal power series:

$$
\mathcal{S}(z)=\sum_{j=2}^{\infty} c_{j} z^{j} \quad(z \in \mathbb{C})
$$

If $M$ is real analytic, then the series $\mathcal{S}(z)$ converges near the origin. But, its converse is not always true. From Cauchy-Hadamard's formula, positivity of the convergence radius of the power series $\mathcal{S}(z)$ is equivalent to the condition lim $\sup _{j \rightarrow \infty}\left|c_{j}\right|^{1 / j}<\infty$. 
Hereafter in this section, we only consider the case when the hypersurface admits a good coordinate at $p$. That is to say, $M$ can be expressed as in the model form

$$
r(z, w, \bar{z}, \bar{w})=2 \operatorname{Re}(w)+F(z, \bar{z})=0,
$$

where $F$ is the same as that in (3.1).

The following theorem gives equivalence conditions for Question 1 in the Introduction in the two-dimensional model case.

Theorem 5.5 Let $M$ be a real hypersurface in $\mathbb{C}^{2}$ admitting a good coordinate at $p$ as in (5.2). If $\Delta_{1}(M, p)=\infty$, then the following three conditions are equivalent.

(i) There exists $a \gamma \in \Gamma^{\mathrm{reg}}$ tangent to $M$ at $p$ to infinite order;

(ii) There exists a good coordinate $(z, w)$ for $M$ at $p$ on which $F$ is flat;

(iii) There exists a good coordinate $(z, w)$ for $M$ at $p$ such that the convergence radius of the power series $\mathcal{S}(z)$ in (5.1) is positive.

Proof (ii) $\Longrightarrow$ (i) and (ii) $\Longrightarrow$ (iii) are obvious.

First, let us show (iii) $\Longrightarrow$ (ii). From (iii), the power series $\mathcal{S}(z)$ can be regarded as a holomorphic function defined on some open neighborhood of $z=0$. Putting $w^{*}=w-\mathcal{S}(z)$, we can express the hypersurface $M$ on the good coordinate $\left(z, w^{*}\right)$ by the equation $2 \operatorname{Re}\left(w^{*}\right)+F^{*}(z, \bar{z})=0$, where $F^{*}(z, \bar{z})=F(z, \bar{z})-2 \operatorname{Re}(\mathcal{S}(z))$. Since $F(z)-2 \operatorname{Re}(\mathcal{S}(z))$ is flat at $z=0$, the existence of coordinate in (ii) is shown.

Next, let us show (i) $\Longrightarrow$ (iii). We may assume that the regular holomorphic curve in (i) can be expressed as $\gamma(t)=(t,-h(t))$ on a good coordinates $(z, w)$ satisfying that $(r \circ \gamma)(t)=O\left(t^{N}\right)$ for every $N \in \mathbb{N}$. Here $h$ is holomorphic near the origin and satisfies $h(0)=0$. Let $\sum_{j=1}^{\infty} a_{j} t^{j}$ be the Taylor series of $h$ at $t=0$, which converges on an open neighborhood of $t=0$. Now, after the first finite sum of the Taylor series of $h$ and $F$ are substituted into the equations $(r \circ \gamma)(t)=-2 \operatorname{Re}(h(t))+F(t, \bar{t})=0$, we have

$$
\operatorname{Re}\left(\sum_{j=1}^{N} a_{j} t^{j}\right)-\sum_{j=2}^{N} \operatorname{Re}\left(c_{j} t^{j}\right)=O\left(t^{N+1}\right)
$$

for every $N \in \mathbb{N}$. From the above equality, we can see that $c_{j}=a_{j}$ for every $j \in \mathbb{N}$. This means that $\mathcal{S}(t)$ converges and $\mathcal{S}(t)=h(t)$ on an open neighborhood of $t=0$.

Remark 5.6 The condition (iii) in Theorem 5.5 is weaker than the condition: $M$ is real analytic near $p$. Indeed, as mentioned in the Introduction, the real analyticity of $M$ implies the existence of desired curves in (i) in the theorem [7,11,19]. The hypersurface $M$ defined by $2 \operatorname{Re}(w)+e^{-1 /|z|^{2}}=0$ with $p=(0,0)$ satisfies the conditions (ii) and (iii), but $M$ is not real analytic at $p$.

In [3,16,22], smooth real hypersurfaces in $\mathbb{C}^{2}$ admitting no curve $\gamma_{\infty}$ in (1.2) are constructed. It follows from Theorem 5.5 that there are many such hypersurfaces.

Corollary 5.7 Let $M$ be a real hypersurface defined by the equation (5.2). If $\Delta_{1}(M, p)=\infty$, then the following two conditions are equivalent. 
(i) There is no $\gamma \in \Gamma$ tangent to $M$ at 0 to infinite order;

(ii) The convergence radius of the power series $\mathcal{S}(z)$ equals zero.

Remark 5.8 From (ii) in the above corollary, it is quite easy to construct smooth hypersurfaces satisfying the condition (i). Let $\left\{c_{j}\right\}_{j \in \mathbb{N}}$ be a sequence of complex numbers such that the convergence radius of $\sum_{j=2}^{\infty} c_{j} z^{j}$ is zero. By using a classical lemma of E. Borel (cf. [21], Theorem 1.5.4, or [14], Theorem 1.2.6), for the formal power series of $(x, y) \in \mathbb{R}^{2}(z=x+i y)$ :

$$
\sum_{j=2}^{\infty} \operatorname{Re}\left(c_{j} z^{j}\right)=\sum_{(j, k) \in \mathbb{Z}_{+}^{2}} C_{j k} x^{j} y^{k} \text { with } C_{j k} \in \mathbb{R},
$$

there exists a real-valued $C^{\infty}$ function $f$ defined near the origin in $\mathbb{R}^{2}$ whose Taylor series at the origin is (5.3). Then the real hypersurface defined by $2 \operatorname{Re}(w)+f(z, \bar{z})=0$ satisfies the condition (i). We remark that the above hypersurface cannot always be uniquely determined from the sequence $\left\{c_{j}\right\}_{j \in \mathbb{N}}$.

In $[16,22]$, similar examples of hypersurfaces have been found. Since their constructions do not use a lemma of E. Borel directly, they look much more elaborate.

Furthermore, it is shown in [9] that when $\left\{c_{j}\right\}_{j \in \mathbb{N}}$ is an increasing sequence of positive real numbers, the above function $f$ can be selected to be a subharmonic function on $\mathbb{C}$. Therefore, there are many pseudoconvex real hypersurfaces satisfying the condition (i) in the corollary.

\section{Higher Dimensional Case}

In this section, we generalize results given in the previous section to the higher dimensional case. Let $M$ be a real hypersurface in $\mathbb{C}^{n+1}(n \geq 1)$ and let $p$ lie in $M$. In this section, we always consider the case when $M$ is of Bloom-Graham infinite type at $p$. First, let us recall the exact definition of the Bloom-Graham type in [3]. We remark that the following definition is an equivalence condition for their original type. This equivalence is also shown in [3].

Definition 6.1 Let $\mathcal{X}$ be a set of $n$-dimensional complex submanifolds containing $p$. We say that the Bloom-Graham type of $M$ is $m(<\infty)$, if there is a $X \in \mathcal{X}$ tangent to $M$ at $p$ to order $m$ but no $X \in \mathcal{X}$ tangent to a higher order. Otherwise, we say that the Bloom-Graham type of $M$ is infinity at $p$ (see the condition (7) in Proposition 1.1).

In the case of Bloom-Graham infinity type, a similar property to Lemma 5.4 can be seen.

Lemma 6.2 If the Bloom-Graham type of $M$ at $p$ is infinity, then the Taylor series of $F$ in (3.1) at the origin consists of only pure terms on every standard coordinate (see Sect. 3).

Proof Let us assume the existence of a standard coordinate $(z, w)$ on which the Taylor series of $F$ contains a mixed term. Let $m$ be the minimum order of the mixed terms of Taylor series of $F$. 
It is easy to construct a new standard coordinate $\left(z, w^{*}\right)$ on which $M$ can be expressed by the equation

$$
2 \operatorname{Re}\left(w^{*}\right)+P_{m}(z, \bar{z})+Q(z, \bar{z})+R_{1}^{*} \cdot \operatorname{Im}\left(w^{*}\right)+R_{2}^{*}=0,
$$

where $P_{m}$ is a non-zero mixed homogenous polynomial of degree $m$ without pure terms, $Q \in C_{0}^{\infty}\left(\mathbb{C}^{n}\right)$ with ord $(Q) \geq m+1$ and $R_{1}^{*}, R_{2}^{*}$ have the same properties as those of $R_{1}, R_{2}$ in (3.1), respectively. It follows from the definition that the BloomGraham type of $M$ at $p$ equals $m<\infty$, which is a contradiction.

From Lemma 6.2, the Taylor series of $F$ at the origin in $\mathbb{C}^{n}$ can be expressed as $2 \sum_{\alpha \in \mathbb{Z}_{+}^{n}} \operatorname{Re}\left(c_{\alpha} z^{\alpha}\right)$ where $c_{\alpha} \in \mathbb{C}$. When the hypersurface $M$ and $p \in M$ are fixed, the sequence $\left\{c_{\alpha}\right\}_{\alpha \in \mathbb{Z}_{+}^{n}}$ is determined by the chosen standard coordinates $(z, w)$. For a given coordinate $(z, w)$, we define the formal power series

$$
\mathcal{S}(z)=\sum_{\alpha \in \mathbb{Z}_{+}^{n}} c_{\alpha} z^{\alpha} \quad\left(z \in \mathbb{C}^{n}\right)
$$

Hereafter in this section, we only consider the case when the hypersurface admits a good coordinate at $p$. That is to say, $M$ can be expressed as in the model form

$$
r(z, w, \bar{z}, \bar{w})=2 \operatorname{Re}(w)+F(z, \bar{z})=0,
$$

where $F$ is the same as in (3.1).

Let $\hat{\Gamma}$ be the set of (germs of) nonconstant holomorphic curves $\hat{\gamma}=\left(\hat{\gamma}_{1}, \ldots, \hat{\gamma}_{n}\right)$ : $(\mathbb{C}, 0) \rightarrow\left(\mathbb{C}^{n}, 0\right)$. Let $\sum_{k=1}^{\infty} a_{j k} t^{k}$ be the Taylor series of $\hat{\gamma}_{j}$ for $j=1, \ldots, n$. After these Taylor series are substituted into $\mathcal{S}\left(z_{1}, \ldots, z_{n}\right)$ with $z_{j}=\hat{\gamma}_{j}(t)$, a formal computation gives the formal power series, denoted by $(\mathcal{S} \circ \hat{\gamma})(t)$, in the following.

$$
\begin{aligned}
(\mathcal{S} \circ \hat{\gamma})(t) & =\mathcal{S}\left(\hat{\gamma}_{1}(t), \ldots, \hat{\gamma}_{n}(t)\right)=\sum_{\alpha \in \mathbb{Z}_{+}^{n}} c_{\alpha} \prod_{j=1}^{n}\left(\sum_{k=1}^{\infty} a_{j k} t^{k}\right)^{\alpha_{j}} \\
& =: \sum_{j=2}^{\infty} c_{j} t^{j} .
\end{aligned}
$$

The relationship between $F$ and $\mathcal{S}$ implies that

$$
(F \circ \hat{\gamma})(t)=2 \sum_{j=2}^{N} \operatorname{Re}\left(c_{j} t^{j}\right)+O\left(t^{N+1}\right),
$$

for every $N \in \mathbb{N}$.

The following theorem is a natural generalization of Theorem 5.6. Let $\Gamma$ denote the set of nonconstant holomorphic mappings $\gamma:(\mathbb{C}, 0) \rightarrow\left(\mathbb{C}^{n+1}, p\right)$.

Theorem 6.3 Let $M$ be a real hypersurface in $\mathbb{C}^{n+1}(n \geq 1)$ admitting a good coordinate at $p$ as in (6.2). If the Bloom-Graham type of $M$ at $p$ is infinity, then the following three conditions are equivalent. 
(i) There exists a $\gamma \in \Gamma$ tangent to $M$ at $p$ to infinite order;

(ii) There exists a good coordinate $(z, w)$ for $M$ at $p$ on which $(F \circ \hat{\gamma})(t)$ is flat at $t=0$ for some $\hat{\gamma} \in \hat{\Gamma}$;

(iii) There exists a good coordinate $(z, w)$ for $M$ at $p$ such that the formal power series $(\mathcal{S} \circ \hat{\gamma})(t)$ in $(6.3)$ converges on an open neighborhood of $t=0$ for some $\hat{\gamma} \in \hat{\Gamma}$, where $\mathcal{S}$ is as in (6.1).

Proof First, let us show (ii) $\Longrightarrow$ (i). Let $\gamma(t)=(\hat{\gamma}(t), 0) \in \Gamma$, where $\hat{\gamma}$ is as in (ii), and let $r$ be a defining function for $M$ as in (6.2). Then we have $(r \circ \gamma)(t)=(F \circ \hat{\gamma})(t)=$ $O\left(t^{N}\right)$ for every $N \in \mathbb{N}$, which implies (i).

Second, let us show (iii) $\Longrightarrow$ (ii). Let $I:=\left\{j: \hat{\gamma}_{j} \not \equiv 0\right\}$. Define the map $T_{I}$ : $\mathbb{C}^{n} \rightarrow \mathbb{C}^{n}$ by $\left(w_{1}, \ldots, w_{n}\right)=T_{I}\left(z_{1}, \ldots, z_{n}\right)$ where $w_{j}=z_{j}$ if $j \in I$ and $w_{j}=0$ otherwise. It follows from Abel's lemma (cf. [12]) that the convergence of $(\mathcal{S} \circ \hat{\gamma})(t)$ for some $t \neq 0$ implies that the power series $\left(\mathcal{S} \circ T_{I}\right)(z)$ converges on an open neighborhood of $z=0$, which means that $\mathcal{S} \circ T_{I}$ can be regarded as a holomorphic function there. Letting $w^{*}=w-\left(\mathcal{S} \circ T_{I}\right)(z)$, we can express the hypersurface $M$ on the good coordinate $\left(z, w^{*}\right)$ by the equation: $2 \operatorname{Re}\left(w^{*}\right)+F^{*}(z, \bar{z})=0$, where $F^{*}(z, \bar{z})=F(z, \bar{z})-2 \operatorname{Re}\left(\left(\mathcal{S} \circ T_{I}\right)(z)\right)$. By using the equality $\mathcal{S} \circ T_{I} \circ \hat{\gamma}=\mathcal{S} \circ \hat{\gamma}$, $\left(F^{*} \circ \hat{\gamma}\right)(t)=(F \circ \hat{\gamma})(t)-2 \operatorname{Re}(\mathcal{S} \circ \hat{\gamma})(t)$ is flat at $t=0$, which implies (ii).

Third, let us show (i) $\Longrightarrow$ (iii). We may assume that a holomorphic curve in (i) can be expressed as $\gamma(t)=(\hat{\gamma}(t),-h(t))$, where $\hat{\gamma} \in \hat{\Gamma}$ and $h \in \mathcal{O}_{0}(\mathbb{C})$ with $h(0)=0$, on a good coordinates $(z, w)$. Note that (i) is equivalent to the condition $(r \circ \gamma)(t)=O\left(t^{N}\right)$ for every $N \in \mathbb{N}$. Let $\sum_{j=1}^{\infty} a_{j} t^{j}$ be the Taylor series of $h$ at $t=0$, which converges on an open neighborhood of $t=0$. Now, after the first finite sum of the Taylor series of $h$ and $F \circ \hat{\gamma}$ in (6.4) are substituted into the equations $(r \circ \gamma)(t)=-2 \operatorname{Re}(h(t))+(F \circ \hat{\gamma})(t)=O\left(t^{N}\right)$ for every $N \in \mathbb{N}$, we have

$$
\operatorname{Re}\left(\sum_{j=1}^{N} a_{j} t^{j}\right)-\sum_{j=2}^{N} \operatorname{Re}\left(c_{j} t^{j}\right)=O\left(t^{N+1}\right),
$$

for every $N \in \mathbb{N}$. From the above equality, we can see that $c_{j}=a_{j}$ for every $j \in \mathbb{N}$. This means that $(\mathcal{S} \circ \hat{\gamma})(t)$ converges and $(\mathcal{S} \circ \hat{\gamma})(t)=h(t)$ on an open neighborhood of $t=0$.

In [9], smooth pseudoconvex real hypersurfaces in $\mathbb{C}^{n+1}$ of Bloom-Graham infinite type admitting no curve $\gamma_{\infty}$ in (1.2) are constructed. It follows from Theorem 6.3 that many such hypersurfaces can be easily constructed.

Corollary 6.4 Let $M$ be a real hypersurface defined by the equation (6.2). If the BloomGraham type of $M$ at 0 is infinity, then the following three conditions are equivalent.

(i) There is no $\gamma \in \Gamma$ tangent to $M$ at 0 to infinite order;

(ii) For all $\hat{\gamma} \in \hat{\Gamma}$, the formal power series $(\mathcal{S} \circ \hat{\gamma})(t)$ does not converge at any point on a delated open neighborhood of $t=0$;

(iii) The formal power series $\mathcal{S}(z)$ does not converge at any point on a delated open neighborhood of $z=0$. 
Proof We remark that (ii) $\Longrightarrow$ (iii) can be shown by using Abel's lemma (cf. the proof of (iii) $\Longrightarrow$ (ii) in Theorem 6.3). The other implications can be directly obtained from Theorem 6.3.

Remark 6.5 From (iii) in the above corollary, it is easy to construct smooth pseudoconvex hypersurfaces of Bloom-Graham infinite type satisfying the condition (i). One of simple examples of hypersurfaces is given by the equation $\operatorname{Re}(w)+f_{1}\left(z_{1}, \bar{z}_{1}\right)+$ $\cdots+f_{n}\left(z_{n}, \bar{z}_{n}\right)=0$, where $f_{j}(j=1, \ldots, n)$ are subharmonic functions constructed in [9] (see also Remark 5.8). The example constructed in [9] takes the same form, but this example needs some additional conditions for each $f_{j}$.

Roughly speaking, when the flatness of hypersurfaces is stronger, it becomes easier to find the curve tangent to $M$ to higher order. Thus, the following question seems to be more difficult: does there exist a smooth pseudoconvex real hypersurface in $\mathbb{C}^{n+1}$ $(n \geq 2)$ of the Bloom-Graham finite type that admits no $\gamma \in \Gamma$ tangent to $M$ to infinite order? The following theorem gives an affirmative answer.

Theorem 6.6 Let $n \geq 2$. There exists a smooth pseudoconvex real hypersurface $M$ in $\mathbb{C}^{n+1}$ with $\Delta_{1}(M, p)=\infty$ and $\Delta_{1}^{\mathrm{reg}}(M, p)<\infty$ (in particular, $M$ is of BloomGraham finite type at $p$ ) that admits no $\gamma \in \Gamma$ tangent to $M$ at $p$ to infinite order.

Proof Notice that since a desired real hypersurface $M$ satisfies that $\Delta_{1}^{\text {reg }}(M, p)<$ $\Delta_{1}(M, p)$, there is no $\mathcal{N}$-canonical coordinate for $M$ from Theorem 2.3.

First, let us construct a desired real hypersurface $M$ in $\mathbb{C}^{3}$. Let $\left\{c_{j}\right\}_{j \in \mathbb{N}}$ be an increasing sequence of positive real numbers such that the power series $\sum_{j=8}^{\infty} c_{j} z^{j}$ does not converge away from the origin. Let $f$ be a real-valued smooth subharmonic function defined near the origin in $\mathbb{C}$ whose Taylor series is $2 \sum_{j=8}^{\infty} \operatorname{Re}\left(c_{j} z^{j}\right)$ (see Remark 5.8). Let us consider the smooth function $F$ defined near the origin in $\mathbb{C}^{2}$ as

$$
F(z, \bar{z})=\left|z_{1}^{3}-z_{2}^{2}\right|^{2}+f\left(z_{1}, \bar{z}_{1}\right)
$$

Let $M$ be a smooth real hypersurface in $\mathbb{C}^{3}$ defined by $r(z, w)=2 \operatorname{Re}(w)+F(z, \bar{z})=$ 0 . The pseudoconvexity of $M$ is obvious.

Now, let us consider $\Delta_{1}(M, 0)$ and $\Delta_{1}^{\text {reg }}(M, 0)$ by using the Newton polyhedron of $\mathcal{N}_{+}(r)$. It is easy to see

$$
\mathcal{N}_{+}(r):=\left\{\xi \in \mathbb{R}_{+}^{3}: \xi_{1} / 6+\xi_{2} / 4+\xi_{3} \geq 1\right\}
$$

Let $\kappa_{0}:=\left\{\left(\xi_{1}, \xi_{2}, 0\right) \in \mathcal{N}_{+}(r): \xi_{1} / 6+\xi_{2} / 4=1\right\}$, which is a compact face of $\mathcal{N}_{+}(r)$. Then $F_{\kappa_{0}}$ is not $\mathcal{N}$-nondegenerate, while $F_{\kappa}$ 's are $\mathcal{N}$-nondegenerate for the other compact faces $\kappa$. Therefore, Theorems 7.3 and 8.4 in [15] implies that if $\gamma \in \Gamma$ does not take the form

$$
\gamma(t)=\left(t^{2}, \pm t^{3}+o\left(t^{3}\right), o\left(t^{12}\right)\right)
$$


then we have

$$
1 \leq \frac{\operatorname{ord}(r \circ \gamma)}{\operatorname{ord}(\gamma)} \leq 6
$$

In particular, every $\gamma \in \Gamma^{\text {reg }}$ does not take the form (6.6), so it is easy to see $\Delta_{1}^{\mathrm{reg}}(M, 0)=6$. More precisely, we consider the case when $\gamma$ takes the form (6.6).

Lemma 6.7 Let $N$ be an arbitrary integer with $N \geq 10$. Then the following two conditions are equivalent.

(i) $\operatorname{ord}(r \circ \gamma) \geq 2 N+2$;

(ii) $\gamma(t)=\left(t^{2}, \pm t^{3}+O\left(t^{N+1}\right),-\sum_{j=8}^{N} c_{j} t^{2 j}+O\left(t^{2 N+2}\right)\right)$.

Proof By dividing the function $r$ into a polynomial part and the remainder part, a defining function for $M$ can be rewritten as follows.

$$
r(z, w)=2 \operatorname{Re}\left(w+\sum_{j=8}^{N} c_{j} z_{1}^{j}\right)+\left|z_{1}^{3}-z_{2}^{2}\right|^{2}+R_{N+1}\left(z_{1}, \bar{z}_{1}\right)
$$

where $R_{N+1} \in C_{0}^{\infty}(\mathbb{C})$ with ord $\left(R_{N+1}\right) \geq N+1$.

First, we show (i) $\Longrightarrow$ (ii). It suffices to treat the curves of the form (6.6), which will be more exactly denoted by $\gamma(t)=\left(t^{2}, \pm t^{3}+g(t), h(t)\right)$ where $g \in \mathcal{O}_{0}(\mathbb{C})$ with $\operatorname{ord}(g)>3$ and $h \in \mathcal{O}_{0}(\mathbb{C})$ with $\operatorname{ord}(h)>12$. Substituting this $\gamma(t)$ into (6.7), we have

$$
(r \circ \gamma)(t)=2 \operatorname{Re}\left(h(t)+\sum_{j=8}^{N} c_{j} t^{2 j}\right)+|g(t)|^{2}+R_{N+1}\left(t^{2}, \overline{t^{2}}\right)
$$

Since $R_{N+1}\left(t^{2}, \overline{t^{2}}\right)=O\left(t^{2 N+2}\right)$ and the mixed terms and the pure terms cannot be canceled, $g$ and $h$ must satisfy that $g(t)=O\left(t^{N+1}\right)$ and $h(t)+\sum_{j=8}^{N} c_{j} t^{2 j}=$ $O\left(t^{2 N+2}\right)$, which imply the condition (ii).

Next, we show (ii) $\Longrightarrow$ (i). Substituting the equation in (ii) into (6.7), we can easily see $\operatorname{ord}(r \circ \gamma) \geq 2 N+2$.

It follows from the above lemma that $\Delta_{1}(M, p)=\infty$.

Now, let us assume that there exists a curve $\gamma_{\infty} \in \Gamma$ such that $\operatorname{ord}\left(r \circ \gamma_{\infty}\right) \geq N$ for every $N \in \mathbb{N}$. Let $\gamma_{\infty}(t)=:\left(\gamma_{1}(t), \gamma_{2}(t), \gamma_{3}(t)\right)$. Since $\gamma_{\infty}$ satisfies the condition (i) in Lemma 6.7, the condition (ii) implies that $\gamma_{3}(t)=-\sum_{j=8}^{N} c_{j} t^{2 j}+O\left(t^{2 N+2}\right)$ for every $N \in \mathbb{N}$. But, $\sum_{j=8}^{\infty} c_{j} t^{2 j}$ does not converge away from the origin, which is a contradiction to the holomorphy of $\gamma_{3}$. As a result, we see that there exists no $\gamma \in \Gamma$ tangent to $M$ at the origin to infinite order.

In higher dimensional case $\mathbb{C}^{n+1}$ with $n \geq 3$, the following $F$ is considered:

$$
F(z, \bar{z})=\left|z_{1}^{3}-z_{2}^{2}\right|^{2}+f\left(z_{1}, \bar{z}_{1}\right)+\sum_{j=3}^{n}\left|z_{j}\right|^{2}
$$


where $f$ is the same as that in (6.5). It is easy to construct higher dimensional hypersurfaces satisfying the properties in the theorem by using $F$ in (6.5) in a similar fashion to the three-dimensional case.

\section{Open Problems}

Theorems 5.5 and 6.3 only treat real hypersurfaces of the model form as in (3.2). The following problem is naturally raised.

Problem 7.1 Let $n \geq 1$ and let $M$ be a general smooth (pseudoconvex) real hypersurface in $\mathbb{C}^{n+1}$ and $p \in M$. Give equivalence conditions for the existence of $\gamma \in \Gamma$ tangent to $M$ at $p$ to infinite order, analogous to those in Theorems 5.5 and 6.3.

The second problem is concerned with Theorem 6.6.

Problem 7.2 Let $n \geq 2$ and let $M$ be a smooth (pseudoconvex) real hypersurface in $\mathbb{C}^{n+1}$ with $\Delta_{1}^{\text {reg }}(M, \bar{p})<\infty$ for $p \in M$. Give equivalence conditions for the existence of $\gamma \in \Gamma$ tangent to $M$ at $p$ to infinite order.

Theorem 6.6 only provides a simple example to the nonexistence of the desired $\gamma \in \Gamma$.

Acknowledgements The author greatly appreciates that Ninh Van Thu kindly informed of important examples of real hypersurfaces of infinite type in [9]. The author also would like to express his sincere gratitude to the referee for his/her careful reading of the manuscript and giving the author valuable comments. This work was supported by JSPS KAKENHI Grant Numbers JP20K03656, JP20H00116.

Open Access This article is licensed under a Creative Commons Attribution 4.0 International License, which permits use, sharing, adaptation, distribution and reproduction in any medium or format, as long as you give appropriate credit to the original author(s) and the source, provide a link to the Creative Commons licence, and indicate if changes were made. The images or other third party material in this article are included in the article's Creative Commons licence, unless indicated otherwise in a credit line to the material. If material is not included in the article's Creative Commons licence and your intended use is not permitted by statutory regulation or exceeds the permitted use, you will need to obtain permission directly from the copyright holder. To view a copy of this licence, visit http://creativecommons.org/licenses/by/4.0/.

\section{References}

1. Arnold, V.I., Gusein-Zade, S.M., Varchenko, A.N.: Singularities of Differentiable Maps I. Birkhäuser, Basel (1985)

2. Arnold, V.I., Gusein-Zade, S.M., Varchenko, A.N.: Singularities of Differentiable Maps II. Birkhäuser, Basel (1988)

3. Bloom, T., Graham, I.: A geometric characterization of points of type $m$ on real submanifolds of $C^{n}$. J. Differ. Geom. 12(2), 171-182 (1977)

4. Catlin, D.: Necessary conditions for subellipticity of the $\bar{\partial}$-Neumann problem. Ann. Math. (2) 117, 147-171 (1983)

5. Catlin, D.: Subelliptic estimates for the $\bar{\partial}$-Neumann problem on pseudoconvex domains. Ann. Math. (2) 126, 131-191 (1987)

6. D'Angelo, J.P.: Real hypersurfaces, orders of contact, and applications. Ann. Math. (2) 115, 615-637 (1982) 
7. D'Angelo, J.P.: Several Complex Variables and the Geometry of Real Hypersurfaces. Studies in Advanced Mathematics. CRC Press, Boca Raton (1993)

8. Fornæss, J.E., Lee, L., Zhang, Y.: Formal complex curves in real smooth hypersurfaces. Illinois J. Math. 58(1), 1-10 (2014)

9. Fornæss, J.E., Ninh, V.T.: A note on pseudoconvex hypersurfaces of infinite type in $\mathbb{C}^{n}$. To appear in Proceedings of the American Mathematical Society arXiv:1804.10087v3

10. Fornæss, J.E., Stensønes, B.: Maximally tangent complex curves for germs of finite type $C^{\infty}$ pseudoconvex domains in $\mathbb{C}^{3}$. Math. Ann. 347, 979-991 (2010)

11. Fornæss, J.E., Stensønes, B.: Infinite type germs of real analytic pseudoconvex domains in $\mathbb{C}^{3}$. Complex Var. Elliptic Equ. 57(6), 705-717 (2012)

12. Fritzsche, K., Grauert, H.: From Holomorphic Functions to Complex Manifolds. Graduate Texts in Mathematics, vol. 213. Springer, New York (2002)

13. Heier, G.: Finite type and the effective Nullstellensatz. Commun. Algebra 36(8), 2947-2957 (2008)

14. Hörmander, L.: The Analysis of Linear Partial Differential Operators. I. Distribution Theory and Fourier Analysis. Second Edition. Grundlehren der Mathematischen Wissenschaften, vol. 256. Springer, Berlin (1990)

15. Kamimoto, J.: Newton polyhedra and orders of contact on real hypersurfaces. To appear in J. Math. Soc. Jpn. arXiv:2004.02475v2

16. Kim, K.-T., Ninh, V.T.: On the tangential holomorphic vector fields vanishing at an infinite type point. Trans. Am. Math. Soc. 367(2), 867-885 (2015)

17. Kolár, M.: Normal forms for hypersurfaces of finite type in $\mathbb{C}^{2}$. Math. Res. Lett. 12(5-6), 897-910 (2005)

18. Kouchnirenko, A.G.: Polyèdres de Newton et nombres de Milnor. Invent. Math. 32, 1-31 (1976)

19. Lempert, L.: On the Boundary Behavior of Holomorphic Mappings, Contributions to Several Complex Variables, vol. 193-215, Aspects of Mathematics, E9, Friedr. Vieweg, Braunschweig (1986)

20. McNeal, J., Némethi, A.: The order of contact of a holomorphic ideal in $\mathbb{C}^{2}$. Math. Z. 250, 873-883 (2005)

21. Narasimhan, R.: Analysis on Real and Complex Manifolds. Reprint of the 1973 edition. North-Holland Mathematical Library, vol. 35. North-Holland Publishing Co., Amsterdam (1985)

22. Nguyen, T.K.S., Chu, V.T.: A note on infinite type germs of a real hypersurface in $\mathbb{C}^{2}$. VNU J. Sci.: Math. Phys. 35(2), 82-87 (2019)

Publisher's Note Springer Nature remains neutral with regard to jurisdictional claims in published maps and institutional affiliations. 